\title{
Factores que protegen la función socializadora de las familias de los estudiantes del colegio La Presentación (Duitama, Boyacá)
}

\author{
Nidia Milena Moreno López ${ }^{1}$
}

\begin{abstract}
Resumen
Hoy en día es muy común escuchar múltiples quejas sobre la disfuncionalidad familiar. Sin embargo son pocos los ejemplos que se tienen sobre aspectos positivos y ejemplos de vida familiar adecuados, funcionales y amorosos en las relaciones intrafamiliares, de familias comunes y corrientes, lejanas a los modelos materialistas presentados por los medios de publicidad, modelos de familias inexistentes por su perfectibilidad, familias que solucionan los problemas por arte de magia. Esta investigación se centró en un grupo de seis familias de estudiantes adolescentes del colegio La Presentación (Duitama, Boyacá), en las que los miembros consideraban poseer bienestar en su contexto familiarSe presentan los factores protectores de las familias que afrontan múltiples problemas de la vida real; quienes de las crisis se fortalecen y funcionan ante las exigencias del medio sociocultural en el que se desenvuelven.
\end{abstract}

Palabras clave: cognición, comunicación, organización, socialización, socioafectividad.

1 Tutora Escuela de Ciencias Sociales Artes y Humanidades, de la Universidad Nacional Abierta y a Distancia -UNAD-. CEAD Yopal. Psicóloga, Especialista en Educación y Orientación Familiar. Candidata Magister en Gerontología Social Universidad de Barcelona, nidia.moreno@unad.edu.co, Colombia. 


\title{
Factors safeguarding the families' socializing function among Duitama (Boyacá) La Presentación school's students
}

\begin{abstract}
Today it is common to hear many complaints about dysfunctional families. However, few examples have positive aspects and examples of proper family life, functional and loving relationships within families, families remote from ordinary material models presented by the advertising media, models exist for their families perfectibility, families solve problems by magic.

This research focused on a group of 6 families of adolescent students of the College of Duitama Presentation, in which members considered to have well in their family context. Identified factors that protect the social function in the processes of communication, affection and partner organization in the process of organization and discipline protective factor, generating habits its members in the communication process models rescuing the importance of providing positive role models to their members and partner in the process of affectivity as a protective factor, the motivation that leads the family in each of its members and as a whole. Protective factors are presented for families facing multiple problems in real life who of the crisis stronger and work with the demands of the sociocultural environment in which they operate.
\end{abstract}

Key words: cognition, communication, organization, socialization, socio-affective

Recibido: 18 de noviembre 2011

Aceptado: 13 de diciembre 2011

\section{Introducción}

El ciclo de la vida familiar atraviesa varias etapas según el momento evolutivo y la interrelación de sus miembros, teniendo en cuenta varios momentos que van desde la conformación de la pareja, pasando por la paternidad joven, padres con hijos en edad preescolar, con hijos de básica primaria, con hijos adolescentes, con hijos jóvenes y adultos, con hijos adultos, hasta el momento en que estos últimos 
conforman a su vez nuevas familias y la pareja inicial comparte nuevamente algunas de las características de la primera etapa al encontrarse sin hijos, momento que finaliza cuando posteriormente acontece la muerte de uno de los cónyuges.

Las etapas anteriores son planteadas en condiciones casi perfectas, porque es sabido que en nuestros tiempos la ausencia de alguno de los dos padres (o ambos) se da desde un comienzo y que las etapas del ciclo evolutivo a nivel sociocultural se han ido alterando; hijos que trabajan, padres analfabetas, familias con solo una cabeza de familia y las familias extensas que han asumido el rol y las funciones de las familias nucleares. Estas son las familias que nuestra cotidianidad presenta y bajo esta premisa es que se hace necesario este estudio para poder, a pesar de sus diferencias, encontrar puntos afines que puedan coadyuvar en el proceso de la orientación familiar para aquí y para ahora, Padilla (1994).

El presente estudio ha tomado familias reconocidas por sus miembros como funcionales, con el objeto de identificar los factores protectores desde los procesos básicos de organización, comunicación y socio afectividad, comprendiendo al individuo en lo emocional, cognitivo y expresivo, buscando modelos de orientación hacia la promoción de la salud familiar ocasionando autorreflexiones familiares frente al quehacer diario y a la potencialidad de acción. El estudio se realizó con familias nucleares de estrato socioeconómico medio-alto del colegio La Presentación de la ciudad de Duitama, se contó con la colaboración y participación de todos los miembros de seis familias que se consideraron así mismas como funcionales; se encontró la disciplina como factor protector del proceso de organización, la motivación desde el proceso de socio afectividad y los modelos desde el proceso de comunicación. Estas familias cumplen con muchas de las características presentes en familias no tan funcionales, sin embargo son capaces de salir triunfantes en la lucha de la vida diaria.

Las familias colombianas en las últimas décadas han venido sufriendo una serie de cambios que les son necesarios para afrontar las exigencias de los nuevos tiempos. Exigencias relacionadas con los estilos de familia, las pautas establecidas para funcionar con padres, madres o hijos ausentes por abandono, secuestro o muerte, por maltrato infantil y/o conyugal reconocido y juzgado severamente por la sociedad, al igual que el abuso sexual. Sin embargo estas transformaciones han surgido en torno a procesos específicos de sus dinámicas familiares que se han mantenido a pesar de las diferencias que existen entre unas y otras ya sea por la misma particularidad en la intimidad familiar o por diferencias marcadas culturalmente. Estos procesos, latentes en las dinámicas familiares, están relacionados con la forma en cómo las familias, están organizadas, cómo han logrado construir su identidad familiar desde sus ritos, creencias y la forma específica de manejar la normatividad y la autoridad familiar, también en la manera en como estas familias han establecido una serie de pautas comunicativas y códigos verbales y no verbales que aseguran su interacción y funcionamiento, así como la construcción de lazos fuertes de convivencia que son traducidos en 
Factores que protegen la función socializadora de las familias de los estudiantes del colegio La Presentación (Duitama,

fuertes y sólidos vínculos afectivos que han logrado permanecer ante las crisis, teniendo en cuenta que las buenas relaciones familiares, como el alto apoyo paterno, la comunicación, la implicación, el control inductivo y la presencia de autoridad facilitan el desarrollo de la identidad (Gecas y Seff, 1990; Kimmel y Weiner, 1998).

Con el estudio de los procesos de las dinámicas familiares de organización, comunicación y socio afectividad que se correlacionan entre sí, se identificaron y reconocieron los factores que han ocasionado el mantenimiento funcional de las familias, que aunque con múltiples diferencias han permanecido unidas $\mathrm{y}$ han logrado adaptarse a los múltiples cambios presentados y los han asumido de mejor forma.

La familia sin duda debe adaptarse continuamente a las diversas novedades que se encuentran en las etapas del ciclo humano y mantenerse en constante equilibrio-desequilibrio. En estas familias el desempeño de las tareas y objetivos básicos es dinámico puesto que en cada etapa la familia asume tareas y estrategias distintas que activan los recursos disponibles, teniendo claro las expectativas, deseos y sueños, como proyecto de vida familiar establecido, y así encontrar pautas generales para la orientación de la familia dentro de su dinámica en su proyecto de vida. Autores como Martínez (2002), clasifican a la familia como insustituible por la característica fundamental en la simultaneidad en las funciones que realiza, cada una es un universo, lleno de características propias que la hacen única e irrepetible, debido a su multiplicidad de funciones, los teóricos las han clasificado en tres ejes esenciales: 1) lo físico y biológico, 2) lo personal, 3) lo social y emocional donde uno y otro eje interactúan entre sí, y que se retoman ya en la investigación en los procesos de organización, comunicación y socio afectividad.

La intencionalidad del proyecto de investigación fue la detección de los factores que están protegiendo a las familias funcionales, y a partir de este reconocimiento poder diseñar e implementar estrategias pedagógicas que permitan orientar de una manera más precisa a los padres y madres de familia, es decir implementar planes de intervención con las familias promoviendo la salud, y no reincidiendo en la enfermedad, proporcionando modelos positivos y funcionales en las dinámicas familiares y así lograr metacognición sobre las pautas transgeneracionales apropiadas. Desde el enfoque sistémico se aborda a la familia con posibilidades desde su estructura y procesos, como un sistema natural, con características propias y que responde a los principios de no sumatividad, con límites, causalidad circular, papeles, reglas y con flexibilidad al cambio interno y externo. La familia, como afirma Rodríguez (2004), es el principal medio de socialización del ser humano, es el órgano encargado de permitirle al individuo un contacto directo con el exterior, así como de garantizar que logre una interrelación armoniosa, o que fracase en este proceso. La familia es en definitiva la que sienta las bases de los valores democráticos 
que han de servir al individuo en su proceso de socialización y que lo van a hacer, o no, más apto en su desarrollo vital. Es importante mencionar el origen de las pautas que la familia genera en su función socializadora, pautas para el desarrollo adecuado del autoconcepto, la autonomía, la responsabilidad, una actitud hacia el compromiso personal, la competencia social, la disposición al esfuerzo personal, la auto superación y el rendimiento; y que se proveen desde los procesos de organización, comunicación y socio afectividad. Entendiendo también al ser humano en sus características propias en el procesamiento de la información desde lo cognitivo, en la manifestación de sus comportamientos desde lo expresivo, y desde la interiorización de sentimientos y emociones desde lo afectivo; en una interrelación de la comprensión del ser con la comprensión de la familia para entenderla y por ende poder extraer los factores protectores de la dinámica de las familias que han favorecido su función socializadora.

\section{Metodología}

\section{Tipo de investigación}

Es un estudio descriptivo de corte etnográfico en el que se busca identificar y describir sistemáticamente hechos y características de una población dada o de un área de interés, tal como se presenta actualmente y con toda exactitud (Forero, 1998:83), en este caso los factores que protegen la función socializadora de las familias en los procesos de organización, comunicación y socio afectividad dentro de las dinámicas familiares.

\section{Construcción de subjetividades a partir de los procesos de socialización}

Esta línea de investigación es con la que se direcciona el trabajo investigativo de la Escuela de Ciencias Sociales Artes y Humanidades de la UNAD, que tiene como objetivo general abordar las dinámicas y problemáticas de orden psicológico desde una perspectiva bio- psico-socio-cultural y comunitaria, contribuyendo al desarrollo de la disciplina y a la construcción de conocimiento desde una perspectiva pluralista e interdisciplinaria que aporte al desarrollo de los individuos y las comunidades. y como objetivos específicos busca indagar los procesos biopsicosociales, culturales y comunitarios que se hacen presentes en las prácticas sociales, así como identificar imaginarios, valores, disposiciones culturales y patrones de organización de contextos que promocionan la conformación de subjetividades y prácticas sociales hacia formas de participación éticas, con conciencia social y reconocer los procesos comunitarios y psicosociales que pueden favorecer la acción transformadora de las problemáticas psicosociales, proponiendo modelos y estrategias para la intervención en problemáticas psicosociales, que favorezcan los procesos participativos autogestionarios y democráticos. 
Factores que protegen la función socializadora de las familias de los estudiantes del colegio La Presentación (Duitama,

De acuerdo a la delimitación espacial, la población objeto de estudio la constituyen familias funcionales de los estudiantes del colegio La Presentación de la ciudad de Duitama.

La muestra la constituyen 6 familias, de estrato socioeconómico medio alto, católicas, con uniones permanentes de 20 años en promedio y de las que sus miembros las consideraron como funcionales en sus dinámicas familiares de los grados noveno y décimo del colegio La Presentación.

\section{Técnicas e instrumentos de recolección de la información}

La encuesta de diagnóstico: Donde se buscaba encontrar las familias que se consideraban así mismas como funcionales, se realizó a través de una breve explicación a los estudiantes de los grados noveno y décimo en cuanto a las características que tenían las familias funcionales aclarándoles que no se buscaban familias perfectas. En esta primera encuesta se seleccionaron 20 familias. Con este instrumento que constaba de 5 preguntas, se cuestionaron aspectos relacionados con la organización, comunicación y socio afectividad, además de su funcionalidad.

La encuesta de análisis: Esta encuesta fue aplicada a 7 de las familias seleccionadas en la encuesta de diagnóstico en razón a que las otras familias se habían retirado del colegio, por el cambio de año. De las familias encuestadas se recibieron y analizaron a 7 familias, ya que entregaron a tiempo la encuesta resuelta y estuvieron dispuestas para la realización de la entrevista familiar. En esta encuesta se realizaron 20 preguntas.

La entrevista: Se realizó con todos los miembros de cada familia, donde se buscaba ampliar la información proporcionada por las entrevistas y así poder categorizar las respuestas de una manera más acertada, teniendo en cuenta la comprensión y vivencia familiar, como la conceptualización teórica de los procesos a investigar.

Categorías de análisis:

Tabla 1. En esta tabla se presentan las categorías que se tuvieron en cuenta en el estudio de las familias y en los individuos.

\begin{tabular}{|c|c|}
\hline En las familias & En los sujetos \\
\hline Organización & Cognición Comunicación \\
Emoción Socio afectividad & Expresión \\
\hline
\end{tabular}


Tabla 2. Identificación de las sub- categorías

\begin{tabular}{|c|c|c|}
\hline Organización & Comunicación & Sociafectividad. \\
\hline $\begin{array}{c}\text { 2. Función de roles Autoridad } \\
\text { Normas Creencias Rituales } \\
\text { Proyectos }\end{array}$ & $\begin{array}{c}\text { Palabras Gestos Miradas } \\
\text { Mímica } \\
\text { Lenguaje Verbal }\end{array}$ & $\begin{array}{c}\text { Emoción Sentimientos Actitudes } \\
\text { Valores Principios Axiologías. }\end{array}$ \\
\hline 3. Metas. & Lenguaje No Verbal. & \\
\hline
\end{tabular}

\section{Resultados}

«Entendemos por socialización el proceso a través del cual el individuo humano aprende e interioriza unos contenidos socioculturales, a la vez que desarrolla y afirma su identidad personal bajo la influencia de unos agentes exteriores y mediante mecanismos procesuales frecuentemente no intencionados» (Coloma Medina, 1993b). En el proceso de organización familiar se presentan varios aspectos que contribuyen en la estabilidad y fortalecimiento de la función socializadora de la familia. Para la investigación se trabajó con familias nucleares, que en sí mismas se consideran como funcionales, pero alejadas de la utopía de la familia perfecta, con uniones de pareja permanente de 20 años promedio, con repertorios de conducta determinados, que son cumplidos por los miembros de las familias a cabalidad, como lo evidencian algunos de los siguientes reportes «siempre se respetan las reglas», «mis padres son los encargados de la autoridad en la casa, dan reglas justas y necesarias», «cada uno sabe sus obligaciones». Estas familias están en una búsqueda constante de satisfacción de necesidades, y para esto se tienen funciones claramente establecidas, en unas el padre es el proveedor económico directo: «todo corre por cuenta de mi papá», «mi papá es quien da las órdenes, es la base más firme», en otras familias son los dos padres quienes dan este soporte «mi padre y mi madre son el soporte económico del hogar». Estas familias se caracterizan por tener definidos dentro de sus dinámicas familiares los valores democráticos como la «honestidad, respeto, unión, tolerancia, humildad, sencillez, trabajo, confianza», además de patrones educativos como la creencia en Dios, el valor de los modelos educativos: «mi hermano mayor es mi ejemplo y el menor mi reflejo», el valor de la familia: «la familia es linda, unida, nos apoyamos unos a otros, es cariñosa, especial, comprensiva y hay amigos». Lo anterior evidencia lo que afirma Rodríguez (2004), la familia es el principal medio de socialización del ser humano, es el órgano encargado de permitirle al individuo un contacto directo con el exterior, encargado de garantizar que ese individuo logre una interrelación armoniosa y positiva del medio o también lo contrario. El manejo de la autoridad gira en torno a la claridad sobre las figuras de autoridad «mis padres tienen absoluta autoridad en el hogar», «mis padres 
Factores que protegen la función socializadora de las familias de los estudiantes del colegio La Presentación (Duitama,

ponen las reglas, yo las cumplo y no peleamos», en el manejo de la autoridad más que perfecto se presenta un conocimiento claro por parte da la familia sobre este, y es asumido, respetado y ejercido por ellos, «mis padres dicen algo claro y se cumple», «los dos juntos toman las decisiones más adecuadas, y que mejor convengan», «en mi familia no son autoritarios pero nadie se cría solo, no son represivos pero nadie es libertino». La toma de decisiones en estas familias aunque es debatida y hasta negociada, son finalmente los padres quienes asumen la responsabilidad de decisión familiar, «se respetan las decisiones de mi mamá y mi papá», «no se desautorizan entre ellos», «concertamos las decisiones, siempre respetando las reglas», «entre mis padres toman los dos las decisiones». Es el proceso mediante el cual las familias fijan reglas de convivencia, extraídas del conjunto de valores, entendidos como el conjunto de creencias perdurables en el tiempo y que orientan el modo de actuar conciente e inconcientemente «un valor es una creencia duradera sobre que un modo específico de conducta o estado final de existenciales preferible personal o socialmente a un modo contrario u opuesto de conducta o estado final de existencia» (Rokeach, 1973:5). Estas familias cuentan con diversos mecanismos de control, restricción o refuerzo del comportamiento de sus miembros y aunque se presentan continuas dificultades y en ocasiones peleas, los sobrellevan con fortaleza, sencillez y humildad. «reinan los sentimientos como el amor, humor y confianza», "es una familia común y corriente, los problemas se solucionan hablando, cuando se necesita exigencia se busca», »es una familia súper, aunque con problemas», y frente a las dificultades consideran como importante, «pedir a Dios se terminen las dificultades», «no desestabilizar el hogar», «plantear soluciones», «llevar a cabo las acciones para ayudar», «ayudarse mutuamente», «hablar sobre lo que sucedió y las posibles alternativas». También se presenta una pedagogía de la independencia y autonomía, «cada uno resuelve sus problemas siguiendo los consejos de papá y mamá». Lo anterior supone un proceso organizado en el cual se encuentran inmersas las pautas que han sido enseñadas por la familia e interiorizadas por cada uno de los individuos durante un contacto continuo y unas formas de interactuar específicas que han generado vínculos estrechos entre ellos fortalecidos y que favorecen el desarrollo del ser humano durante su ciclo vital. Representados en una adecuada vivencia de los valores democráticos.

La disciplina se concibe en este estudio como uno de los factores protectores desde el proceso de la organización familiar, y que hace referencia a la capacidad de actuar ordenada y perseverantemente para conseguir un bien. Exige un orden y unos lineamientos para poder lograr más rápidamente los objetivos deseados Cotera (2009).

Dentro del proceso de organización se detectó que la disciplina genera hábitos en sus miembros, el alcance en la satisfacción de necesidades básicas y meta necesidades, se genera estímulos agradables que potencian la conducta $y$ estímulos desagradables que debilitan conductas inadecuadas, además de 
ocasionar de manera más directa el manejo de situaciones frustrantes y la capacidad de afrontar situaciones difíciles, logrando un adecuado manejo interno preparado para asumir el éxito con sencillez y el fracaso con fortaleza. Se encontraron como necesarios la restricción a algunas cosas o situaciones, el refuerzo a eventos positivos, el control como apoyo en la moderación de la conducta, la necesidad de establecer patrones educativos claros y alcanzables, con roles establecidos para el cumplimiento de responsabilidades, el manejo de la voluntad generando conductas básicas de autocontrol, la negociación y sobre todo la generación de los valores democráticos en todos y cada uno de sus miembros con responsabilidad social. En este sentido la actitud de los padres a menudo consiste en negociar con el adolescente. Las relaciones entre padres y adolescentes conciernen también a la participación en la vida del grupo doméstico, su lugar en las decisiones. Las referencias que tienen los padres de su propia adolescencia también pueden servir para guiar a sus hijos. Si bien las actitudes de los jóvenes manifiestan una gran innovación en todo lo relativo a las libertades personales, están marcadas, en cambio, por una gran conservación de los valores y de las normas.

En las familias se encontró una delicada utilización del lenguaje en su quehacer diario, lenguaje verbal y no verbal como: «todos procuramos solucionar los problemas y salir adelante juntos», «cuando lo necesitamos, dialogamos mucho, razonamos los errores y las cosas que no hacemos bien», «hablamos para hacer lo que es lo mejor, y no hacer lo que no nos conviene». En la comunicación intrafamiliar hay una clara manifestación de los valores, como la confianza «yo les cuento a mis papás, que mis amigas no creen capaz entre ellas, por ejemplo: los muchachos que me molestan, cosas así». Lo que muestra que los padres como primeros cuidadores, en una situación «suficientemente» buena, establecerán un vínculo, una sintonía con el niño/a que les permitirá interpretar aquellas demandas de atención y de cuidado que precise su hijo en cada momento. Ellos serán los primeros responsables en la creación de unos canales y significación que favorecerán la construcción de la identidad del niño. López (1995:9). La familia es para el niño su primer núcleo de convivencia y de actuación, donde irá modelando su construcción como persona a partir de las relaciones que allí establezca $\mathrm{y}$, de forma particular, según sean atendidas sus necesidades básicas (Brazelton y Greenspan, 2005). Se participa en la toma de decisiones, «se da alguna idea y todos participan, hasta el punto de tomar una decisión». Existe una constante comunicación, «no solo con palabras, con sentimientos, miradas, afecto y comprensión». Hay una escucha activa con una actitud de respeto y apoyo mutuo, «siempre hablamos cuando hay problemas», «no nos juzgamos, nos escuchamos mutuamente, hablamos mucho y nos contamos los problemas». También se evidencia una actitud mutua de interés por el otro, «nos sentamos y hablamos, es bacano porque todos sabemos lo que piensan los unos de los otros», además de ver la disposición de las familias en la escucha, «mi papá nos reúne a todos en la sala y lo resolvemos todo con el diálogo». Se focaliza en el problema «se le da valor a las cosas importantes y no 
Factores que protegen la función socializadora de las familias de los estudiantes del colegio La Presentación (Duitama,

a las que no la tienen». Los conflictos son manejados en la medida de lo posible en torno a la «tranquilidad», «respeto», «amor», «sinceridad», «tolerancia» , «apoyo», «comprensión», «paciencia», «unión a la hora de tomar decisiones en beneficio común»; con todas las orientaciones anteriores se considera el gran valor que tienen los modelos en el aprendizaje y se determina como el segundo factor protector. Según Barudy (2005) los buenos tratos a niñas y niños aseguran el buen desarrollo y el bienestar infantil y son la base del equilibrio mental de los futuros adultos $\mathrm{y}$, por tanto, de toda la sociedad.

Hay una constante expresividad afectiva, sea para apoyar o para restringir comportamientos y para garantizar la expresión y conductas adecuadas, «nos demostramos el amor con hechos, y con las actitudes que reflejamos», se evidencian sentimientos como: «cariño, amor, alegría, humor», con expresiones desde las palabras, los abrazos, los besos; se presenta confianza en la expresión de sentimientos como la «angustia, la tristeza». Las demostraciones afectivas son continuas, especialmente en fechas importantes o en situaciones difíciles, «nos decimos que nos queremos, nos apoyamos en momentos difíciles», «cuando necesito palabras de aliento o un sentimiento de afecto lo pido y ellos me lo brindan», las expresiones de afecto no son limitadas a la edad o jerarquía, «mis papás me consienten resto, en ocasiones parezco una niña chiquita, y me divierto mucho jugando con mis hermanitos menores», «mis papás entre ellos son muy cariñosos, lo expresan a cada rato con palabras y abrazos». El afecto es dado como un reforzador primario, «nos premian con su afecto de padres y hermanos, no es como en otras familias que se premia con dinero». El optimismo y la motivación son una constante para seguir adelante, superar el fracaso, los obstáculos y disfrutar los logros, «mis padres son muy afectuosos, siempre están motivándonos y animándonos». Las actitudes más importantes para estas familias están directamente relacionadas con los valores democráticos, con la «solidaridad, ánimo, apoyo, compañía, preocupación, interés por el otro, colaboración». También se encuentra una clara disposición para la realización de actividades en familia, se orienta en el alcance de objetivos, hay constancia y persistencia de la conducta frente al alcance de metas y proyectos planeados, se evidencian actitudes de liderazgo, se planean actividades, el error es tomado como impulso para mejorar, es valorado el «esfuerzo, responsabilidad, espiritualidad, crecimiento personal, la confianza y el trabajo»; al igual que el optimismo: «hemos tenido muchos obstáculos, pero siempre hemos salido adelante».

Ante las dificultades las familias manifiestan «tristeza, preocupación», pero con una actitud positiva, se piensa en el «consejo, apoyo, o ayuda que se pueda recibir», reconociendo sus limitaciones. Estos reportes son una muestra clara de que la afectividad es una realidad compleja, referida a un conjunto de estados afectivos, sentimientos, emociones e intereses, valores que posteriormente contribuyen a crear una rama más del sistema, relacionándose con otras personas en otras situaciones Satir (1997). Surge entonces la motivación, como el tercer factor protector de la dinámica familiar, se percibe el logro en la conformación 
de un sistema de valores propio, que permite entender, sentir y juzgar la realidad de un modo personal; este aspecto guarda estrecho vínculo con el sentido crítico referido en la autonomía intelectual, pues la participación de ambas será requisito ineludible para que la persona piense por cuenta propia y piense correctamente" (Pérez, Cánovas y Gervilla, 1999: 59).

En el proceso de socio-afectividad, se detectó que la familia es origen de motivación en cada uno de sus integrantes para la realización de sus actividades cotidianas. Cuando hay una orientación para el alcance de objetivos propuestos, la familia es un punto de apoyo para la persistencia, dedicación e intensidad para el logro de esas metas. Así mismo, las pautas relacionadas con la profesión de fe, generan modelos de liderazgo, que son interpretados por los miembros de la familia como actitudes de seguridad y autoestima, lo que permite afrontar los errores como una oportunidad para mejorar y para el cambio. De esta forma se encuentra que los individuos se sienten parte del grupo familiar, así como queridos, respetados y comprendidos, cuando en la familia hay demostraciones de apoyo y ánimo continuos, en dónde ese respeto se manifiesta a pesar de las diferencias, y sin exaltar las debilidades, en un entorno en que el sacrificio y la frustración enderezan el carácter, cuando en la familia se celebra la vida y se mantiene un buen estado de ánimo en la mayoría de las ocasiones, a pesar de los problemas y dificultades, mostrando una actitud optimista frente la vida.

Con todo lo anterior se amplía la visión comprensiva con respecto a los factores protectores frente a la función socializadora de la familia, y se puede determinar que para su análisis es necesario tener en cuenta el conjunto de procesos que intervienen en la comprensión del ser humano, entendidos estos desde la relación individuo-familia-sociedad.

Se concluye que en la interrelación continua entre familia-individuo e individuo- familia surgen tres factores que protegen la función socializadora de la familia: la disciplina, los modelos y la motivación; en donde la vida de la familia depende de la compresión de los sentimientos y necesidades subyacentes a los acontecimientos familiares cotidianos Satir (1988). Estos factores actúan como el motor de impulso frente al alcance de sus metas y proyectos y en donde las estrategias motivacionales, son actividades que sirven para crear y mantener un ambiente adecuado, y están dirigidas a que el estudiante desarrolle y mantenga un estado interno apropiado que le permita establecer metas y concentrarse e indagar sobre su desempeño en la vida (Berroterán, 2010).

\section{Conclusiones}

En el proceso de organización se reveló la importancia que tiene para los padres de familia ser claros y concisos a la hora de instaurar las reglas y 
Factores que protegen la función socializadora de las familias de los estudiantes del colegio La Presentación (Duitama,

normas que serán la guía para el manejo de la autoridad y donde los valores democráticos están presentes en la dinámica de estas familias a través de los procesos de organización, comunicación y socio-afectividad. Las reglas impuestas deben ser adaptables al contexto familiar y en esa medida deben ser exigidas y cumplidas, así mismo, los rituales encaminados a la celebración de fechas especiales, como la vida, logros, alcance de metas y proyectos, son un aliciente afectivo para las familias y se constituyen como pre disponentes para el fortalecimiento del proceso de socio-afectividad.

El fracaso y el error deben ser tomados como un motor de cambio e impulsador hacia el mejoramiento de los miembros y de las relaciones familiares, y el comportamiento de los sujetos guía o modelos de las familias adquieren un gran valor en la medida en que son imitados de una u otra manera por sus miembros.

El lenguaje es un promotor de cambio, de mejora y de sostenimiento familiar, en la medida que permite la cercanía afectiva, el traspaso cultural y potencia la resolución asertiva de los conflictos en congruencia con modelos en la adquisición de normas y reglas.

\section{Referencias bibliográficas}

Berroterán, Yamilis. 2010. «Estrategias Motivacionales dirigidas a docentes para el fomento de la orientación vocacional en estudiantes». en Monografías.com Extraido de internet el 2 de junio de 2011 en http://www.monografias.com/trabajos 88/ estrategias-motivacionales-fomento-orientacion-vocacional/estrategiasmotivacionales-fomento-orientacion-vocacional.shtml

Chance, P. 1994. Aprendizaje y conducta. México: Manual Moderno.

Davidoff, L. 1989 Introducción a la psicología, Bogotá: Mac Graw Hill.

Davis, F. 1996. La comunicación no verbal. Traducción de Rita Mourglier; Madrid: Alianza.

Durkheim, E. 1990. Educación y pedagogía, consejos y controversias, Bogotá: Icfes.

Fernández - Dols, J.M., J. Iglesias y M.J. Mallo. 1992. Comportamiento no verbal y emoción, Madrid: Alhambra.

Forero, Gilberto. 1998. Nuevos Horizontes Pedagógicos, Tunja: UPTC.

Gross, R. 1994. La ciencia de la mente y la conducta. México: Manual Moderno. 
Martínez, Eduardo. «Los elementos del sistema cultural». Extraido de internet el 15 de mayo de 2010 en http://www.monografias.com/trabajos16/autoestima-motivacion/ autoestima-motivacion.shtml\#familia.

Mir, M., M. Batle y M. Hernández.2009. «Contextos de colaboración familia-escuela durante la primera infanci»a. en Revista Electrònica d'Investigació i Innovació Educativa i Socioeducativa, V.1:1:45-68. Consultado en http://www.in.uib.cat/pags/ volumenes/vol1_num1/m-mir/index.html en (mayo 6 2011).

Páez, D., M. Villarreal, A. Etxeberría yJ. Valencia. 1987. Cognición social: esquema y función cognitiva aplicada al mundo social, Madrid: Fundamentos.

Papalia, Diane y Sally Wendkos Olds. 1992. Psicología del desarrollo de la infancia.

Rodríguez, Miguel Ángel. 2004. Un análisis de la educación familiar desde la teoría pedagógica: propuesta de bases fundamentales para un modelo integrado. Universitat de Valencia, Server Publicacions.

Sally; W. 1998. Desarrollo humano, Bogotá: Mac Graw-Hill.

Sprenger, Jacques. «Discipline Starts at Home». Extraído de internet el 2 de junio de 2011 en http://ezinearticles.com/?Discipline-Starts-at-Home\&id=1691324

Vallejo, J. 1991. Introducción a la psicopatología y psiquiatría. Barcelona: Salvat. 